\title{
Migración de trabajo calificado e isomorfismo en sectores empresariales de las principales ciudades mexicanas
}

\author{
Skilled labor migration and isomorphism in business sectors \\ of the main Mexican cities
}

\section{Amilcar Orlian Fernández Domínguez}

Universidad Autónoma de Chihuahua, México

Recibido el 04 de noviembre de 2015; aceptado el 25 de abril de 2017

Disponible en Internet el 19 de marzo de 2018

\section{Resumen}

Recientemente los aportes referentes a la teoría organizacional apuntan a la importancia de la evolución simbiótica entre las organizaciones y su ambiente. El presente estudio aborda esta relación considerando la migración de trabajo calificado como un elemento ambiental que incide en la evolución de organizaciones empresariales, manifestada en un isomorfismo. A su vez, el isomorfismo es medido por dos indicadores: el nivel de diversificación de los sectores económicos y el número de empresas certificadas con estándares de calidad. Se estima el efecto de una mayor migración de personas con educación superior en los indicadores de isomorfismo para 78 ciudades mexicanas mediante modelos econométricos, utilizando variables instrumentales para controlar la endogeneidad de la migración. Los resultados dan evidencia de que la migración de trabajo calificado incide en ambos indicadores, pero se requiere pasar un umbral para que influya en el isomorfismo de las empresas en las ciudades mexicanas.

Códigos JEL: D21, J24, O15, R23.

Palabras clave: Ambiente organizacional, Isomorfismo organizacional, Migración, Trabajo calificado.

Correo electrónico: afernand.uach@gmail.com

La revisión por pares es responsabilidad de la Universidad Nacional Autónoma de México 


\begin{abstract}
Recently, the contributions regarding the organizational theory highlight the importance of the symbiotic evolution between organizations and their environment. This study addresses this relation considering skilled labor migration as an environmental element that influences the evolution of business organizations, manifested, shown in isomorphism. In turn, isomorphism is measured by means of two indicators: the level of diversification of the economic sectors, and the number of companies certified with quality standards. The effect of greater migration of people with higher education in the indicators of isomorphism for 78 Mexican cities is estimated through econometric models, using instrumental variables to control endogeneity of the migration. The results provide evidence that skilled labor migration has an effect on both indicators, but it is necessary to cross a threshold so that it influences the isomorphism of the companies in the Mexican cities.
\end{abstract}

JEL Classification: D21, J24, O15, R23.

Keywords: Organizational environment, Organizational isomorphism, Migration, Qualified labor.

\title{
Introducción
}

Desde el enfoque de evolución de las organizaciones expuesto en la obra de Hannan y Freeman (1977) se ha investigado el impacto de factores ambientales en la diferenciación de éstas como proceso de supervivencia. Sin embargo, a partir de Powell y Di Maggio (1999) comenzó una serie de investigaciones que parten de la premisa de que el ambiente provoca que las organizaciones agrupadas en determinados campos sostengan un isomorfismo, es decir, un proceso de homogeneización respecto a sus estructuras organizacionales.

La literatura aborda diversos factores causantes de isomorfismo entre los cuales se encuentran la reformas a la legislación contable (Doadrio, Alvarado y Carrera, 2015), la injerencia de agencias reguladoras (Moura, Domingos, Cabral y Santos 2014; Filho y Sousa, 2013), la implementación de prácticas ambientales (Gellers, 2012; Morais, Olveira y Souza, 2014; Borges, Dutra y Scherer, 2014), la cultura (Gallén y Peralta de Grado, 2016; Fuentes, 2013), el sistema legal general, la gobernabilidad y la globalización (Gallén y Peralta de Grado, 2016), la participación en el índice de sustentabilidad Dow Jones (Jacomossi, Casagrande y Reis, 2015), el financiamiento extranjero (Mir y Bala, 2015; Salas, 2010), y los clientes, trabajadores y sindicatos (Filho y Sousa, 2013). Como es evidente, el papel de la fuerza laboral en la homogeneización organizacional ha sido estudiado muy poco.

En relación con la fuerza laboral, una variable de especial interés como uno de los mecanismos con los que el ambiente incide en las organizaciones es la migración, pues altera la fuerza laboral disponible, y por tanto determina en cierto grado su evolución y estructura (Manic, 2016; Castelhano, Lawell, Sumner y Taylor, 2016; Mora Rivera y Arellano González, 2016; Santermer \& Giangaspero, 2011; Nava-Tablada, 2012; Chen, Frank, Thomas y Jianguo, 2012). En el caso de México hay pocos estudios que abordan el isomorfismo (Sánchez Ramos, 2007; Ruiz Ortega, 2015; Lara Gómez y Pérez Sosa, 2015; Rivera y González, 2016; Cabrera Rubio, 2016; Rosas, Sánchez y Martínez, 2012); y aunque Gonzáles Zepeda y Escala Rabadán (2014) analizan el caso de migrantes michoacanos, no estiman a la migración como factor de isomorfismo. En este sentido, considerando la importancia histórica y reciente de la inmigración nacional e internacional en México, y que la teoría organizacional resalta que el ambiente funge como una fuerza simbiótica en el desarrollo de las organizaciones, surge el 
interés por investigar sobre el papel de la inmigración de trabajo calificado en el isomorfismo de la estructura empresarial de las principales ciudades mexicanas.

De esta forma, el presente estudio continúa en la segunda sección abordando aspectos teóricos sobre las dos dimensiones de análisis: el ambiente organizacional y la migración. De la teoría organizacional trasciende por una parte el argumento de que el ambiente y la organización se afectan mutuamente (hay endogeneidad), y por otra (junto a algunas teorías de migración discutidas en la sección 2.2), que la evolución de ambos se puede ver reflejada en un isomorfismo causado por la fuerza laboral altamente calificada en búsqueda de una mayor competitividad. Posteriormente, en la tercera sección se plantea la metodología empleada para analizar empíricamente el efecto de la migración de trabajo calificado en el isomorfismo de los sectores económicos de setenta y ocho ciudades mexicanas controlando la endogeneidad de la migración. En la cuarta sección se muestran los resultados, y finalmente la quinta sección brinda algunas conclusiones.

\section{Aspectos teóricos}

\section{Ambiente organizacional}

Recapitulando que los aportes de la administración científica y la escuela del comportamiento no bastaron para explicar la complejidad concerniente al ámbito organizacional, muchos teóricos comenzaron a adoptar una postura de la organización como un sistema abierto, es decir, en la que el ambiente incide en cierta manera en el desempeño y función de la organización. De acuerdo a la teoría de la contingencia, el ambiente establece ciertas restricciones y condiciones incidentes en el funcionamiento de las organizaciones, lo cual requiere cierta capacidad para adaptarse a las particularidades del entorno cambiante. Mintzberg (1997) argumenta que la teoría de contingencia se queda incompleta al tratar de explicar el desempeño organizacional en términos de los factores externos e internos que afectan a la organización, por lo que propone incluir el funcionamiento conjunto de cuatro elementos primordiales: las partes de una organización, sus mecanismos de coordinación, los parámetros de diseño, y los factores situacionales, entre los que se encuentra el ambiente.

Los argumentos de la teoría de la contingencia concernientes a que cierto tipo de organizaciones puede tener un buen funcionamiento únicamente en determinados ambientes ubican a la organización en una postura de adaptación ambiental. Esta postura fue contrastada con la teoría de ecología poblacional de Hannan y Freeman (1977) quienes argumentan que una teoría completa de la organización y su ambiente debe considerar tanto la adaptación como la selección, pues no son aspectos excluyentes sino que se complementan. En este sentido, estos autores parten de la cuestión central de que el ambiente optimiza combinaciones de organizaciones (racionalidad de la selección natural), y esta selección involucra la competencia y el principio de isomorfismo; por tanto, plantean un modelo ecológico de competencia que define el proceso de crecimiento de la población en función de, por una parte, la capacidad del ambiente de soportar formas de organización, y por otra, de la tasa a la que la población cambia cuando el ambiente soporta cambios. Morgan (2006) argumenta que esta postura determinista ha sido criticada por minimizar la importancia de la administración estratégica; al respecto Mintzberg, Ahlstrand, y Lampel (1998) exponen algunas críticas a la ecología poblacional en general sobre el papel del origen de las variaciones ambientales y a las acciones estratégicas agresivas de otra organización. 
Morgan (2006) argumenta que la teoría de contingencia se basa en el supuesto de que el ambiente y la organización son independientes; sin embargo, otras teorías refutan este supuesto, como la teoría de ecología poblacional de Hannan y Freeman (1977) o la teoría del caos, la cual supone a la organización y al ambiente como elementos del mismo patrón interconectado, caracterizados por múltiples sistemas e interacciones ordenados y caóticos que presentan disturbios aleatorios y que, a su vez, producen eventos y relaciones impredecibles. Rivas Tovar (2009) resalta que de acuerdo a Cambell (1993), esta teoría del caos se caracteriza por ser dinámica, no lineal y con elementos transaccionales. Otras teorías suponen una relación más compleja entre la organización y el ambiente, como la teoría de la dinámica de sistemas lejanos al equilibrio de Prigogine y la teoría de sistemas complejos adaptativos. Burgelman (2015) menciona que Prigogine juzgaba que la evolución de un sistema social implicaba una interacción en el comportamiento de sus actores y la incidencia restrictiva aleatoria del ambiente; y por su parte, la teoría de adaptación al borde del caos considera que los sistemas complejos se ubican entre el orden y el caos, y son un resultado natural de la evolución selectiva.

Si bien hasta este punto las teorías expuestas han reconocido la existencia de una relación bidireccional entre el ambiente y la organización, el papel de las características de la fuerza laboral toma importancia con la teoría institucional abordada por Powell y Di Maggio (1999). Aunque dicha teoría se basa en el modo en que las reglas formales e informales inciden en las organizaciones, los autores mencionan que el concepto de isomorfismo es más complejo en el sentido de que una organización es inducida (por factores ambientales) a parecerse a las demás con el fin de adaptarse al contexto ambiental, y éste puede ser reflejado en características organizacionales relacionadas al mercado (isomorfismo competitivo) o a las reglas, políticas o legislación (isomorfismo institucional); bajo el concepto de isomorfismo, las organizaciones empiezan un proceso de homogeneización al buscar innovar con el propósito de lograr un mejor desempeño o un cambio organizacional.

Powell y Di Maggio (1999) enfatizan que las instituciones causan un isomorfismo organizacional a través de la burocratización y mediante mecanismos ambientales coercitivos, miméticos y normativos, siendo este último tipo donde destacan la profesionalización y la presión de la fuerza laboral altamente capacitada. El isomorfismo normativo se da por dos razones: primeramente, porque el perfil profesional es similar en los trabajadores altamente calificados, dada la similitud en las características de sus conocimientos adquiridos (a su vez originado por el isomorfismo existente en las organizaciones de educación profesional); y en segundo término, por la socialización o trascendencia de las redes profesionales fuera de la organización. Además, dado que la homogeneización se puede dar en la estructura, proceso y/o conducta de los grupos o campos organizacionales, los indicadores de isomorfismo variarán dependiendo del campo y objeto de análisis, aunque sugieren observar a la desviación estándar de las variables a estudiar. De esta forma, considerando que la migración está estrechamente vinculada a la cantidad y cualidad de la fuerza laboral en una localidad, la teoría detrás del concepto de isomorfismo justifica la importancia de analizar la migración de trabajo altamente calificado como factor ambiental en la evolución de las organizaciones productivas de esas localidades.

\section{Migración}

De acuerdo a Massey (2015), la teoría de migración gira en torno a elementos 
socioeconómicos e institucionales relacionados con los lugares de origen y destino, así como con las características de los individuos propensos a migrar y a las organizaciones fomentadoras de flujos migratorios; diversas teorías relacionan estos flujos con el ambiente organizacional y/o con la cualidad del trabajo migratorio. La teoría económica neoclásica propone la idea de que al haber mayor fuerza laboral en una localidad que en otra, las diferencias en el producto marginal del trabajo y por tanto del salario provocan un desplazamiento de trabajo (inmigración de trabajadores) hacia el lugar donde pagan más; el desplazamiento causa que el producto marginal disminuya y por lo tanto el salario, hasta alcanzar un equilibrio. Los modelos HarrisTodaro y Todaro-Maruszko extienden esta teoría, argumentando el primero que la decisión de migrar está más bien en función de la brecha de ingresos esperados entre el lugar de origen y destino, y el segundo se basa en los retornos vitalicios del trabajo. Esta teoría implica que la migración tiene un efecto de crecimiento en la industria (al aumentar la producción), pero no asume ninguna especificación sobre la cualidad del trabajo migrante.

Bajo la teoría de capital social, la migración se explica por la creación de vínculos o redes sociales establecidas por migrantes pioneros, quienes al adquirir conocimiento y experiencia respecto a los gastos y riesgos inherentes a la migración, permiten que dichos costos y riesgos para futuros migrantes se reduzca, incentivándolos a migrar (Massey, 2015). Por otra parte, la teoría institucional y la teoría de sistemas mundiales explican la migración a través de la injerencia que imponen los cambios en la estructura de los mercados, la legislación, normas y reglas formales e informales, en la población de un lugar, provocando que cierto segmento de éste (como los trabajadores altamente capacitados) se vuelva propenso a migrar (Massey, 2015). Ahora, de acuerdo a la teoría económica convencional de Piore (1979), la migración se da porque las industrias establecen mecanismos para sostener las jerarquías de trabajo con los salarios más bajos a través de programas de trabajo migrante temporal auspiciados por los gobiernos o por ellas mismas Así, estas teorías por tanto diferencian la fuerza laboral migrante de acuerdo a su cualidad, pero se enfocan en el efecto de la industria hacia la migración.

La posible diferencia en la cualidad de la fuerza migrante se expone nuevamente en la teoría de la nueva economía de migración laboral expuesta por Stark y Bloom (1985), en la cual la migración se origina en los sentimientos de los individuos hacia la percepción que tienen de su privación o satisfacción relativa con sus ingresos o habilidades, por tanto la decisión de migrar generalmente se toma hacia dentro de un grupo afectado por tal decisión, ya sea la familia o un grupo social más complejo, con el fin de reducir los costos y riesgos (mediante remesas e ingresos poco correlacionados con la fuente de ingresos obtenidas en el lugar de origen), y aprovechar el capital de redes y parentescos (Goldin, Reinert y Beath, 2007). Con relación a lo anterior se encuentra la aplicación de la teoría financiera de portafolios (Anam y Chiang, 2007), en la cual se argumenta que la familia diversifica su portafolio de activos (miembros familiares) movilizando a alguno de sus miembros a distintos mercados laborales con el fin de aumentar los ingresos totales y reducir el riesgo.

Otra teoría reciente expuesta por Massey (2015) es la de la crisis migratoria, en la que los flujos migratorios se explican por intereses y comportamientos de burócratas, políticos y expertos, quienes movilizan recursos políticos y materiales con fines aparentemente no correlacionados con la migración. De acuerdo con esta teoría, la migración se "sataniza", al relacionarla con conceptos negativos como crimen o ilegalidad; los actores políticos emplean la solución del "problema" como medio para lograr otros objetivos. Esto es muy diferente a lo planteado por la teoría del estado, en la que las organizaciones de empleadores y trabajadores 
persuaden a actores políticos y gobierno para restringir o ampliar el flujo de migrantes primordialmente por cuestiones económicas (Massey, 2015); en momentos de auge económico, el desempleo es bajo y la producción elevada, lo que motiva a las organizaciones empleadoras a solicitar un mayor número de inmigrantes. En estos casos la política migratoria es flexible en el sentido de que pueden limitarse las características de la fuerza migratoria en términos de, por ejemplo, geografía o nivel de capital humano. De especial interés se encuentra el estudio de la migración de trabajadores calificados o personas con capital humano alto (con educación superior), pues se considera que las empresas y organizaciones tienen una injerencia primordial en la determinación de este grupo de migrantes (Pekkala Kerr \& Kerr, 2013; Bermúdez Rico, 2010; Rodríguez Gómez, 2009) y viceversa.

Si bien estas teorías se enfocan primordialmente en los determinantes de la migración, resultan evidentes algunos aspectos importantes en relación con los objetivos de este estudio. Por una parte, distintas teorías evidencian diferencias sobre el contexto en que se presentan los flujos migratorios de trabajadores calificados y no calificados; por tal motivo, es de esperarse que sus efectos en las organizaciones sean asimismo diferentes, y por tanto es justificable realizar un análisis que aborde un tipo de migrantes en particular, en este caso, los altamente calificados. Por otra parte, aunque algunas teorías no plantean efectos específicos de la migración en la organización, dejan entrever sus efectos generales en la industria a través de efectos en la producción o competitividad. Al respecto, la literatura muestra claramente que la migración es un fenómeno de gran importancia por sus efectos tanto para el lugar de origen como para el de destino (Peri, 2012; Tovar \& Vélez, 2007; Boustan, Fishback, \& Kantor, 2010; Hjorth, 2009; Ortega \& Peri, 2009; Goldin et al., 2007), entre los cuales resaltan los efectos en la estructura de sectores organizacionales productivos. Por ejemplo, aunque algunos estudios muestran que las remesas se destinan primordialmente al consumo (Zarate-Hoyos, 2004; Cuong y Mont, 2012; Airola, 2007) hay evidencia de que la migración puede fomentar el desarrollo de organizaciones productivas en el lugar de origen a través de la inversión de remesas (Manic, 2016; Castelhano, Lawell, Sumner y Taylor, 2016; Mora Rivera y Arellano González, 2016; Santermer \& Giangaspero, 2011) o, a menos que exista desempleo estructural que permita el "reemplazo" de dichos trabajadores, limitarlo mediante la disminución de la fuerza laboral (Nava-Tablada, 2012; Miluka, Carletto, Davis \& Zezza, 2007). Un último aspecto a resaltar es la existencia de una relación causal bidireccional posiblemente compleja (no lineal) entre la migración y las organizaciones, es decir, las dos se explican mutuamente y por tanto se trata de variables endógenas; esta situación puede presentar sesgos al tratar de estimar el efecto de la migración en las variables organizacionales si no es aislado el efecto inverso.

\section{Metodología}

Para estimar el efecto que tiene un factor ambiental en el isomorfismo organizacional, independientemente del efecto de otros factores ambientales, primeramente se realiza un análisis estadístico de las variables de interés, para posteriormente adecuar un modelo econométrico de regresión multivariable. Para ello, las variables propuestas en el análisis se toman con base en la teoría o la evidencia empírica de estudios previos. Respecto a la variable dependiente relativo al isomorfismo, Powell y Di Maggio (1999) mencionan que éste se refleja en características organizacionales relacionadas con el mercado (isomorfismo competitivo) o con las reglas, políticas o legislación (isomorfismo institucional); por lo tanto, se propone 
realizar la estimación de dos variables dependientes correspondientes a estos dos tipos de isomorfismo. Asimismo Powell y Di Maggio (1999) mencionan que la unidad de análisis del isomorfismo puede ir más allá de las empresas que compiten entre sí, abarcando incluso a la totalidad de los actores importantes; así, el campo organizacional analizado en este estudio es el de la totalidad de empresas establecidas en las setenta y ocho principales ciudades mexicanas.

De acuerdo con Hannan y Freeman (1977) citados por Powell y Di Maggio (1999), el isomorfismo competitivo puede medirse por cambios en los nichos de mercado en general; esto abarca desde modificaciones en la estructura empresarial en un campo específico como en grupos de sectores económicos agregados. Así, se puede investigar si una disminución en el número de sectores económicos en una ciudad está influido por mecanismos de isomorfismo competitivo que buscan, por ejemplo, aprovechar las economías de escala en una industria mediante su concentración en una localidad. Por otra parte, como se mencionó anteriormente, el isomorfismo institucional puede medirse por variables relativas a cambios en políticas organizacionales; así, se propone medir este tipo de isomorfismo a través de la proporción de empresas que adoptan una política de calidad o sustentabilidad manifestada por la adquisición de una certificación ISO 9000 o ISO 14000. Respecto a la variable explicativa, se busca identificar si una mayor inmigración de personas calificadas (trabajadores potenciales) incide en un isomorfismo competitivo o institucional de las empresas establecidas en una ciudad determinada, medido por una convergencia en la obtención de certificaciones o un menor número de sectores económicos.

De acuerdo con la teoría econométrica, para obtener un estimador confiable de la variable independiente de interés es importante incluir variables de control pertinentes. Con base en la teoría y en la evidencia empírica referida en la primera sección, estas variables incluyen fuerzas relativas al grado de dependencia de la organización concernientes a factores externos. Powell y Di Maggio (1999) argumentan que la dependencia y la imitación a otras organizaciones son mecanismos coercitivos y miméticos de isomorfismo, por lo que se incluye el número de grandes empresas que se encuentren en el ranking de CNN-Expansión de las 500 empresas más importantes de México (empgrand) por considerarse líderes; además dado que refieren al isomorfismo como un proceso en el que las organizaciones cambian en respuesta a los cambios de otras en el mismo campo, es importante controlar los valores pasados de la variable dependiente (la certificación o la diversificación). Del mismo modo, Powell y Di Maggio (1999), Mir y Bala (2015) y Salas (2010) sugieren que una mayor dependencia al financiamiento externo genera un mayor isomorfismo, por lo que se incluyen el monto de créditos otorgado a las empresas en pesos (creditos) y la inversión extranjera directa neta (ied). También se incluye una variable de productividad por estar vinculado con mecanismos de isomorfismo como la injerencia de agencias reguladoras, trabajadores y sindicatos (Filho y Sousa, 2013), la cultura (Fuentes, 2013), y la implementación de prácticas ambientales (Gellers, 2012). Finalmente se incluye una variable de la población con educación superior (pobeducsup) debido a que Powell y Di Maggio (1999) argumentan que la profesionalización es un mecanismo normativo de isomorfismo.

\section{Modelo econométrico}

Finalmente, el tema de la endogeneidad se trata econométricamente mediante variables instrumentales, es decir, variables no correlacionadas con la variable dependiente, pero sí con la variable endógena explicativa (Wooldridge, 2009). Cuando hay datos disponibles en el tiempo, 
como en el caso de datos de panel longitudinal, la estrategia es utilizar los rezagos de las variables como instrumentos de ellas mismas (Cameron y Trivedi, 2010). Así, para estimar el efecto de la migración de trabajo calificado en las dos variables de isomorfismo empresarial controlando la endogeneidad de la variable migratoria, se propone el modelo econométrico (1):

$$
v d_{i t}=\alpha+\beta m t c_{i t}+C_{i t}{ }^{\prime} \gamma+\varepsilon_{i t}
$$

donde el indicador de isomorfismo $(v d)$ es expresada por el número de sectores económicos (diversec) basado en el Sistema de Clasificación Industrial de América del Norte (SCIAN) o el número de empresas con certificación ISO 9000 e ISO 14000 (eciso) por cada mil empresas de la entidad i en el tiempo t. Para el indicador de migración de trabajo calificado ( $m t c$ ) se utiliza como variable el número de migrantes mayores de 25 años con educación superior en miles $(m i g c)$ o la proporción de estos migrantes con relación al total de la población mayor de 25 años (pmigc) de la entidad i en el tiempo t. $\mathrm{C}$ es el vector de variables de control explicada en la sección anterior, $\varepsilon$ es el error idiosincrático, $\alpha$ y $\beta$ son parámetros, y $\gamma$ es un vector de parámetros.

\section{Datos}

Partiendo de las características de la unidad de análisis (ciudades mexicanas) y que las variables de interés están vinculadas al ámbito de competitividad, se tomaron los datos "brutos" utilizados por el Instituto Mexicano para la Competitividad (IMCO) en la construcción del índice de competitividad urbana (IMCO, 2016) los cuales incluyen datos anuales (2008-2014) de setenta y ocho ciudades consideradas en dicho índice. Cabe mencionar que por falta de disponibilidad de datos a nivel ciudad de la variable eciso (número de empresas certificadas con ISO 9000 y 14000), se agruparon los datos de las ciudades por entidad federativa, de manera que se reportan datos estatales anuales (2008-2013) en las estimaciones de este modelo. Las propiedades de (1) y la disponibilidad de datos para varias ciudades por varios años permiten que se realice la estimación de un modelo econométrico dinámico con datos de panel longitudinal mediante el método generalizado de momentos en dos etapas de Arellano-Bond (Cameron y Trivedi, 2010).

\section{Resultados}

La Figura 1 muestra el número de clases de sectores económicos en las setenta y ocho ciudades consideradas en el análisis. De esta gráfica primeramente se puede observar que las principales ciudades del país (México, Monterrey y Guadalajara) por su tamaño tienen una gran diversificación de clases económicas, cerca de 900²; por otra parte, las ciudades con menos diversidad promedio fueron Río Verde-Ciudad Fernández y Guanajuato (poco menos de 360 clases económicas). Otro dato interesante es que en la mayoría de las ciudades (56 de las 78 analizadas) disminuyó el número de sectores económicos entre 2012 y 2013, indicando

${ }^{2}$ De acuerdo al INEGI, el total de sectores económicos del SCIAN es de 20, los cuales se desagregan en 1051 clases. 
una menor diversidad; desagregando el análisis por niveles, esta disminución estuvo presente mayormente en aquellas ciudades con poco más de 600 sectores económicos.

El hecho de que las ciudades con mayor diversidad sean aquellas que tienen también mayor población, aunado a que la mayoría tuvo una disminución en el número de sectores económicos a partir de 2013, puede plantear el problema de enfrentarse con una relación espuria al argumentar que la migración de personas calificadas está correlacionada positivamente con el tamaño de la localidad a donde migran; sin embargo, al observar la Figura $2^{3}$, es claro que no existe una relación positiva constante entre el número de sectores y la proporción de migrantes calificados, sino que aumenta a una tasa decreciente.

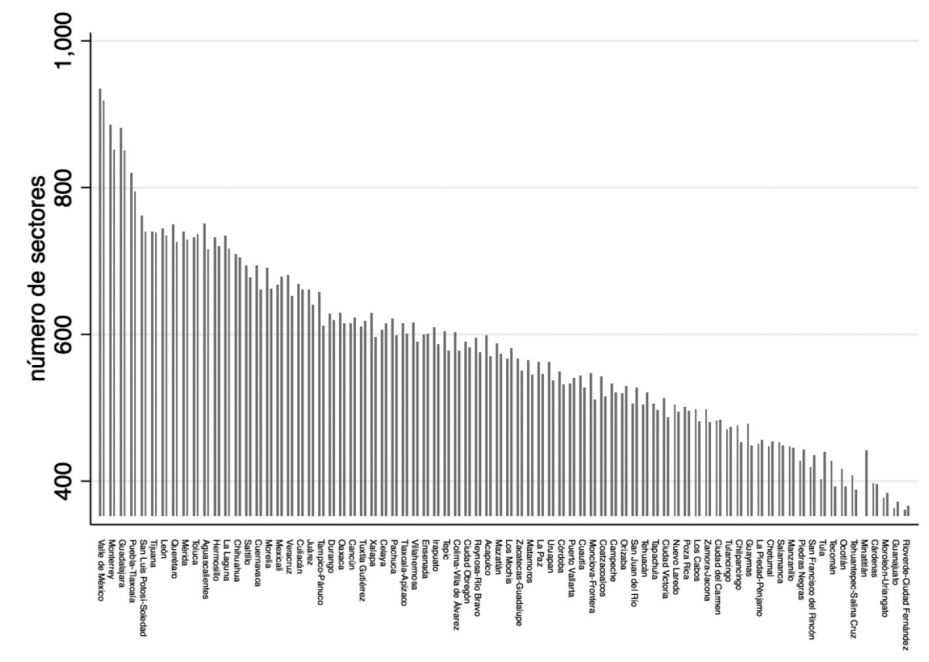

Figura 1. Diversificación de sectores económicos por ciudad 2012-2013. Fuente: elaboración propia con datos del IMCO (2016).

De la misma forma, es evidente que el promedio y la desviación estándar de los sectores disminuyó durante el periodo analizado (cuadrante I de la Figura 3), lo cual de acuerdo a Powell y Di Maggio (1999) sugiere la presencia de isomorfismo. Por su parte, el número de migrantes calificados y su proporción con relación al total de población con el mismo rango de edad (25 años o más) muestran un aumento sostenido en el periodo observado, con una ligera caída en la proporción de migrantes calificados entre 2013 y 2014. De esta figura resalta la relación inversa existente entre ambas variables en el tiempo, es decir, mientras el número de migrantes y su proporción aumentaron, el número de sectores económicos se redujo; sin embargo, aunque esta relación inversa parece respaldar la hipótesis de que la migración de trabajo calificado causa isomorfismo, es necesario realizar un análisis adecuado de causalidad mediante el modelo econométrico.

Partiendo de la relación hallada en la Figura 2, se estimó el modelo (1) primeramente analizando si había evidencia de una relación positiva lineal, y luego permitiendo un

\footnotetext{
${ }^{3}$ Aunque sólo se muestra la relación para el 2014 en esta figura, la misma relación se presenta en los otros años.
} 
comportamiento cuadrático. En los modelos 1 y 2 de la Tabla 1 se tiene a migc como variable independiente, y en el resto a pmigc; la forma funcional de los modelos 1,3 y 5 permite un efecto lineal de la migración, mientras que los modelos 2, 4 y 6 suponen una relación cuadrática; los modelos 3 y 4 prueban si la significancia de pmigc es robusta a pesar de la correlación elevada (coeficiente de correlación de Pearson de .99) de pobeducsup con la variable de población mayor de 25 años utilizada en la construcción de pmigc. Finalmente cabe mencionar que se hicieron pruebas para comprobar el cumplimiento de los supuestos de consistencia en los estimadores de Arellano-Bond y de sobreidentificación válida de Sargan, resultando todos los modelos adecuados al 95\% en ambas pruebas; por otro lado, dado que los errores estándar reportados normalmente presentan sesgo (Cameron y Trivedi, 2010), se utilizan errores estándar robustos, los cuales además resuelven la heteroscedasticidad potencial en el error idiosincrático.

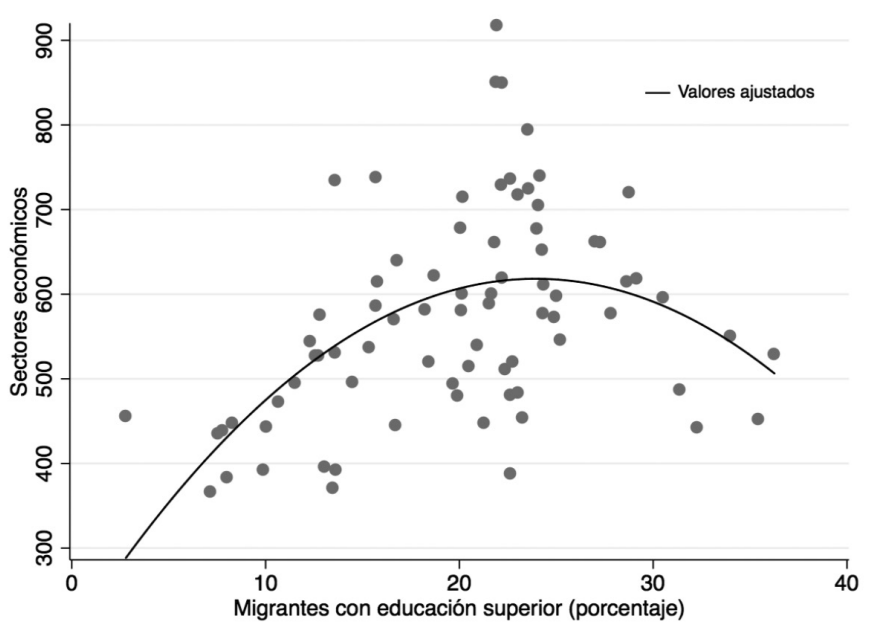

Figura 2. Sectores económicos y migrantes calificados en ciudades mexicanas (2014) Migrantes calificados como proporción del total de personas mayores de 25 años. Fuente: elaboración propia con datos del IMCO (2016).

La Tabla 1 muestra que el coeficiente de créditos a las empresas presenta signo diferente dependiendo de si se incluye en el modelo el número o proporción de migrantes calificados; cuando se incluye el número de migrantes, el coeficiente de créditos tiene signo positivo, contrario a la teoría, pero sólo es significativo en el modelo 2 (aunque no aparecen todos los decimales por espacio, se requiere cerca de doscientos mil millones de pesos de crédito a las empresas para que se forme un sector económico más). En el caso de los modelos donde se estima la proporción de migrantes, el signo del coeficiente de créditos a las empresas es negativo conforme a la teoría pero sólo significativo al $95 \%$ en los modelos 5 y 6; además, el efecto es nuevamente minúsculo. Estas discrepancias cuestionan el papel de los créditos en la diversificación económica de las ciudades, e invitan a realizar un estudio más profundo sobre las características de estos créditos como los mecanismo de otorgamiento, las características de los adquirientes, y el destino real de los recursos. Por ejemplo, es posible que una gran proporción de estos créditos se destine a empresas pequeñas que ofrecen productos o servicios 
tradicionales como preparación de alimentos (restaurantes) o salones de belleza, los cuales pertenecen a sectores económicos que presentan pocos o nulos cambios en el tiempo, además de que requieren poca innovación en sus procesos y por tanto tienen poca o nula injerencia en la diversidad de otros sectores económicos.
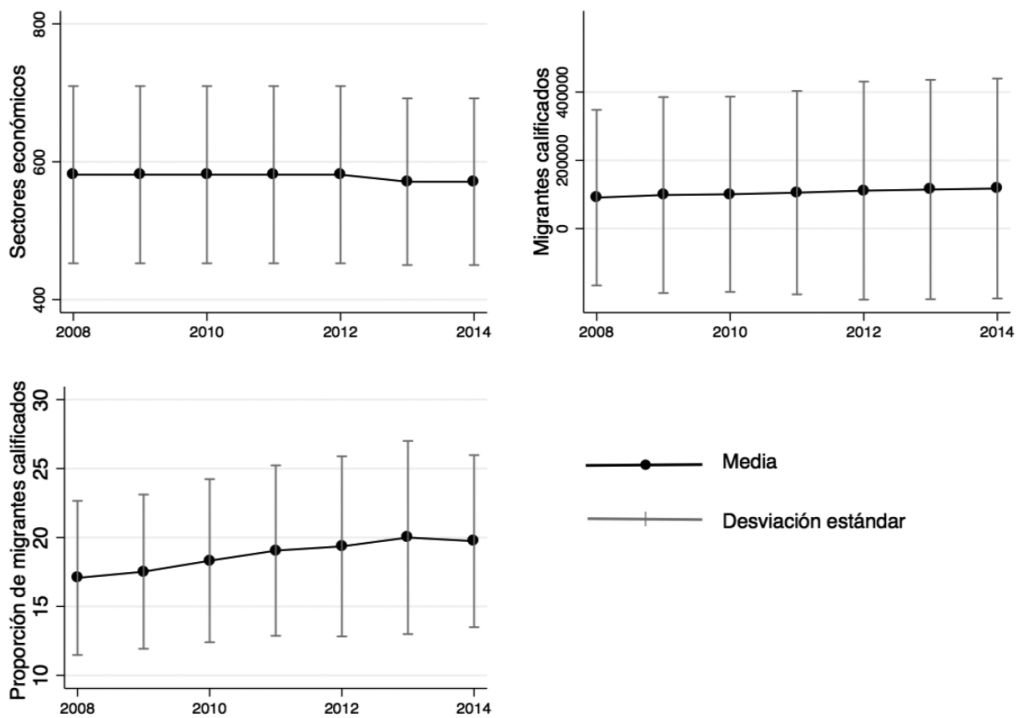

Figura 3. Evolución de los sectores económicos y migrantes calificados en ciudades mexicanas (2008-2014). Promedios y desviaciones estándar correspondientes al total de ciudades.

Fuente: elaboración propia con datos del IMCO (2016).

Se observa en la Tabla 1 que el coeficiente de inversión extranjera directa (IED) tiene el signo esperado y es significativo en los primeros cuatro modelos, indicando que aproximadamente un aumento de cien millones de dólares de IED conduce a una reducción de 1 o 2 sectores económicos en promedio. Este resultado evidencia que los sectores económicos en las ciudades mexicanas tienen una mayor dependencia (como campo organizacional) hacia la IED que a los créditos otorgados localmente, posiblemente por ser destinados en mayor proporción a proyectos de investigación y desarrollo, lo que se traduce en un mayor isomorfismo. Finalmente es indudable que la última variable de control incluida (empresas grandes) tiene un efecto en el isomorfismo, pues es significativa en todos los modelos; sin embargo, presenta inconsistencia al tener signo esperado sólo en los modelos 5 y 6 de la Tabla 1, cuando no se controla por la población con educación superior. Evidentemente existe una alta correlación entre las variables de empresas grandes y la población con educación superior (correlación de Pearson de .98), lo cual dificulta aislar el efecto parcial de ambas variables. 
Tabla 1

Estimación de (1) utilizando como variable dependiente el número de sectores económicos en la ciudad (diversec).

\begin{tabular}{|c|c|c|c|c|c|c|}
\hline diversec & 1 & 2 & 3 & 4 & 5 & 6 \\
\hline diversec (L1) & $0.1960 * *$ & $0.2145 * *$ & $0.3703 * *$ & $0.3606 * *$ & $0.6281 * *$ & $0.5961 * *$ \\
\hline pobeducsup & $-0.6787 * *$ & $-0.6266 * *$ & $-0.2198 * *$ & $-0.2227 * *$ & & \\
\hline productividad & -0.0076 & -0.0127 & -0.0015 & -0.0012 & -0.0200 & $-0.0514 *$ \\
\hline creditos & 0.0000 & $0.0000 * *$ & -0.0000 & -0.0000 & $-0.0000 *$ & $-0.0000 *$ \\
\hline ied & $-0.0161 * *$ & $-0.0125 * *$ & $-0.0210 * *$ & $-0.0210 * *$ & -0.0076 & -0.0073 \\
\hline empgrand & $10.9407 * *$ & $9.4044 * *$ & $6.9084 * *$ & $7.1448 * *$ & $-1.7745 * *$ & $-1.8130 * *$ \\
\hline migc & $0.4520 * *$ & $0.3526 *$ & & & & \\
\hline migcsq & & 0.0000 & & & & \\
\hline pmigc & & & 0.2939 & 0.2849 & $-1.6350 * *$ & $1.8104 * *$ \\
\hline pmigcsq & & & & -0.0001 & & $-0.0875 * *$ \\
\hline $\mathbf{N}$ & 370 & 370 & 370 & 370 & 370 & 370 \\
\hline chi2 & 147.56 & 453.07 & 297.22 & 297.43 & 324.01 & 343.95 \\
\hline p-value & 0.00 & 0.00 & 0.00 & 0.00 & 0.00 & 0.00 \\
\hline
\end{tabular}

leyenda: * $\mathbf{p}<.05 ; * * \mathbf{p}<.01$

Fuente: elaboración propia con datos del IMCO (2016).

En relación con los estimadores de la variable de interés, de los modelos 1 y 2 de la Tabla 1 se observa que la variable de número de migrantes calificados presenta un signo positivo y no hay evidencia estadística de que la relación sea cuadrática. Este coeficiente explica que si el número de migrantes calificados en una ciudad aumenta en aproximadamente dos mil individuos, se espera que aumente un sector económico; en otras palabras, no hay evidencia de que un mayor número de migrantes conduzca a un isomorfismo como se argumentó a raíz de la Figura 3. Sin embargo, los argumentos planteados en las figuras 2 y 3 sobre el efecto de la proporción de migrantes calificados en el número de sectores económicos se respaldan con los modelos 5 y 6 . El coeficiente de la proporción de migrantes en el modelo 5 indica que un aumento de 1 punto porcentual induce una disminución de 1.6 sectores económicos en promedio (hay isomorfismo conforme a lo esperado). Por su parte, la especificación del modelo 6 es la única con todos los coeficientes significativos y con el signo esperado; en este caso se indica que conforme aumenta la proporción de migrantes con educación superior, el número de clases de sectores económicos en una ciudad aumenta a una tasa decreciente, es decir, hay evidencia de un umbral en esa proporción (aproximadamente 10.34\%) a partir de la cual una mayor proporción de migrantes calificados en una ciudad incide en una disminución en los sectores económicos, es decir, en un isomorfismo. De esta forma, parece haber una conciliación con los hallazgos de los modelos 1 y 2 en el que se encuentra un efecto positivo en el efecto de un número mayor de migrantes: para que haya isomorfismo, no basta con que haya mayor inmigración de trabajadores calificados, pues la población de la ciudad aumenta naturalmente; es necesario que estos trabajadores calificados inmigrantes pasen un umbral de modo que representen la mayoría en relación con las demás personas calificadas. Estos resultados tienen sentido bajo el argumento de que conforme mayor sea el grupo de trabajadores con cierto perfil ideológico, más fácil se permea dicha ideología a los demás trabajadores, lo cual es básicamente la justificación detrás del isomorfismo normativo expuesta por Powell y Di Maggio (1999). 
Continuando con el análisis del isomorfismo institucional, la Figura 4 muestra algo similar a la Figura 1, en el sentido de que las ciudades o entidades más importantes económicamente son las que cuentan con mayor número de empresas certificadas con ISO. Además, se observa que aunque el número de estas empresas aumentó anualmente en todas las entidades, es bastante notoria la cantidad relativamente baja de empresas que obtienen esta certificación en las principales ciudades mexicanas, donde el promedio sólo es de aproximadamente 71 por cada mil que hay en la ciudad. Ahora, la relación entre el número de empresas certificadas y los migrantes con educación superior se muestra en la Figura 5, la cual parece sustentar la hipótesis del estudio al existir una clara relación positiva entre ambas variables en cada año.

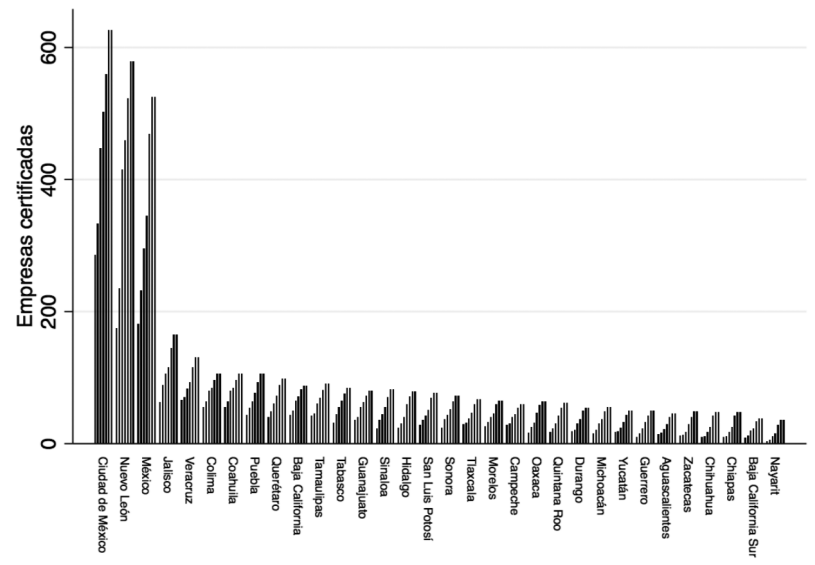

Figura 4. Empresas con certificación ISO 9000 e ISO 14000 por entidad. Número de empresas certificadas por cada mil anual (2008-2014).

Fuente: elaboración propia con datos del IMCO (2016).

Así, las estimaciones del modelo (1) considerando como variable dependiente el logaritmo del número de empresas certificadas con ISO 9000 e ISO 14000 se muestran en la Tabla $2^{4}$. En los modelos 1 y 2 se estimó el número de migrantes calificados; en los modelos 3 y 4 se estimó la proporción de migrantes calificados. Los resultados de la Tabla 2 indican que los modelos se estimaron con datos para las 32 entidades del país por cuatro años (por la pérdida de información al utilizar los instrumentos); además, todos los modelos fueron estadísticamente significativos en conjunto. Casi todas las variables fueron significativas individualmente, aunque algunas presentan el signo contrario a lo esperado, pues de acuerdo a la discusión teórica sobre isomorfismo se espera que un aumento en éstas ocasione un número mayor de empresas certificadas.

Así, la Tabla 2 muestra primeramente que la dinámica de la variable dependiente en el tiempo es significativa y positiva, es decir, el aumento en el número de empresas certificadas con ISO 9000 e ISO 14000 en determinado año tendrá un efecto positivo casi en la misma magnitud en el número de empresas certificadas el año siguiente; este resultado era evidente

\footnotetext{
${ }^{4}$ Nuevamente se hicieron las pruebas de consistencia de Arellano-Bond y de sobreidentificación válida de Sargan, resultando todos los modelos adecuados al $99 \%$ en ambas pruebas.
} 
en la Figura 4 donde claramente se aprecia el aumento sostenido en el número de empresas certificadas para todas las entidades del país. En este caso por tanto, se puede afirmar que el isomorfismo institucional sigue una retroalimentación del mismo proceso de homogeneización, es decir, existe un "contagio" mediante el cual la presencia de empresas certificadas causa que otras empresas busquen y obtengan dicha certificación.

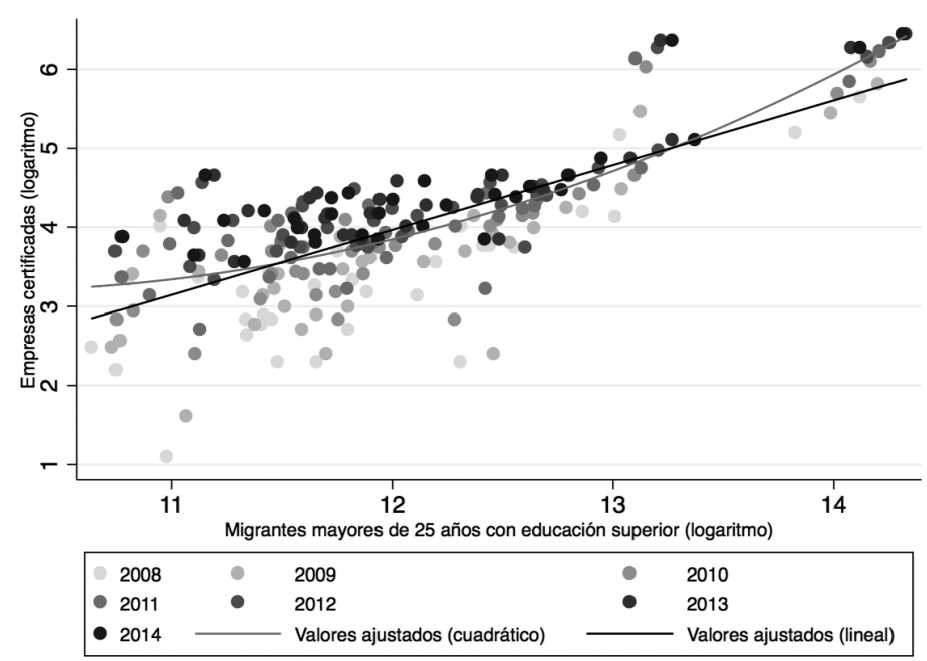

Figura 5. Migrantes con educación superior y empresas certificadas con ISO 9000 e ISO 14000 Datos estatales para los años 2008-2014.

Fuente: elaboración propia con datos del IMCO (2016).

Los modelos de la Tabla 2 muestran que un aumento de la población con educación superior tiene un efecto negativo en la participación de empresas certificadas por cada mil. Si el aumento en la población con educación superior se da únicamente por la gente local (no migrante), se requieren 50 mil habitantes calificados más para que el número de empresas certificadas por cada mil disminuya en $1 \%$, y considerando que ese promedio es menor de cien empresas (Figura 4), entonces se requerirían mucho más habitantes para que se reduzca una empresa certificada; ahora si se permite la inclusión de migrantes con educación superior, se requeriría un aumento en la población capacitada de cerca de 20 mil personas para que se dé dicha reducción. El efecto negativo de la variable pobeducsup hacia la variable de empresas certificadas puede explicarse porque los cambios en este segmento de la población estén vinculados a un cambio desproporcionado en la relación empresas certificadas-empresas totales, es decir, es posible que una mayor población con educación superior aumente tanto el número de empresas totales como el número de empresas certificadas, pero la velocidad en la que aumenta el número de empresas totales es mayor (debido al tiempo que tarda conseguir una certificación ISO y/o a que algunas empresas no puedan solventar los costos), lo cual reduce el valor de la variable eciso a pesar de que realmente haya más empresas certificadas. 
Tabla 2

Estimación del modelo (1) utilizando como variable dependiente el número de empresas certificadas con ISO 9000 e ISO 14000 (eciso).

\begin{tabular}{|c|c|c|c|c|}
\hline & 1 & 2 & 3 & 4 \\
\hline eciso (L1) & $0.9116 * *$ & $0.9354 * *$ & $0.9323 * *$ & $0.9282 * *$ \\
\hline pobeducsup & $-0.0002 * *$ & $-0.0007 * *$ & -0.0001 & -0.0001 \\
\hline ied $(\log )$ & $-0.1192 * *$ & $-0.1344 * *$ & $-0.1313 * *$ & $-0.1393 * *$ \\
\hline créditos (log) & $0.0489 * *$ & $0.0453 * *$ & 0.0358 & 0.0339 \\
\hline productividad & $0.0020 * *$ & $0.0026 * *$ & $0.0024 * *$ & $0.0025 * *$ \\
\hline empgrand & $-0.0001 * *$ & $-0.0001 *$ & $-0.0001 * *$ & $-0.0001 * *$ \\
\hline $\operatorname{migc}(\log )$ & $0.1517 * *$ & $-0.9456^{*}$ & & \\
\hline $\operatorname{migcsq}(\log )$ & & $0.1096 * *$ & & \\
\hline pmigc & & & -0.0008 & 0.0064 \\
\hline pmigcsq & & & & -0.0001 \\
\hline $\mathbf{N}$ & 128 & 128 & 128 & 128 \\
\hline chi2 & 2100744 & 1565064 & 5901490 & 7477345 \\
\hline p-value & 0.00 & 0.00 & 0.00 & 0.00 \\
\hline
\end{tabular}

Leyenda: * $\mathbf{p}<.05 ; * * \mathbf{p}<.01$

Fuente: elaboración propia con datos del IMCO (2016).

Un efecto similar mostrado en la Tabla 2 es el del aumento en la inversión extranjera directa y el número de empresas grandes, las cuales ante un aumento de $1 \%$ en la IED o de una empresa grande se da una disminución en el número de empresas certificadas con ISO 9000 e ISO 14000 en aproximadamente $0.12 \%$ o $0.01 \%$ respectivamente. Nuevamente, la explicación se basa en la relación que pueda tener la IED o el establecimiento de empresas grandes con la razón empresas certificadas-empresas totales: es posible que el flujo de IED o el establecimiento de una empresa grande tenga un impacto menor en la certificación de las empresas existentes.

Aunque tuvo el signo esperado conforme a la teoría en todos los modelos, el crédito a las empresas sólo fue significativo cuando se mantuvo constante el número de migrantes calificados (modelos 1 y 2 de la Tabla 2), y su efecto fue pequeño: un aumento del crédito a las empresas de una ciudad de $1 \%$ incrementa en promedio el número de empresas certificadas en $0.05 \%$. Al contrario de lo sucedido con la IED y el establecimiento de grandes empresas, es comprensible que esta variable tenga el efecto esperado dado que el recurso es susceptible de destinarse al pago de la certificación que a la creación de nuevas empresas. Asimismo la productividad presentó el signo esperado y fue muy significativa en todos los modelos; en este caso, un incremento de la productividad en 1 punto porcentual en promedio presenta un incremento de $0.25 \%$ en el número de empresas certificadas.

Los modelos de la Tabla 2 muestran que el número y la proporción de migrantes calificados en una ciudad tienen un efecto acorde a la teoría en ambas formas funcionales (lineal y cuadrática); sin embargo, la proporción de migrantes no fue significativo (modelos 3 y 4), indicando que en el caso de las empresas certificadas es más bien relevante la presencia de migrantes calificados sin importar cuántos trabajadores calificados no migrantes haya en la ciudad. Ahora, cuando se modela de forma lineal el número de migrantes calificados (modelo 1), la estimación muestra que un aumento de ellos incrementa en promedio el número de empresas certificadas en .15\%; esto en sí es interesante y respalda la hipótesis del estudio sobre el efecto de la migración de trabajo calificado en el isomorfismo y lo hallado en la Figura 5. Además, el modelo de forma 
cuadrática (modelo 2) complementa la información señalando que si el número de migrantes calificados es bajo en una ciudad, un aumento de estos migrantes provoca una disminución en el número de empresas certificadas por cada mil en casi la misma magnitud, posiblemente por su efecto mayor en la creación de nuevas empresas; sin embargo, cuando la ciudad ha pasado un umbral de migrantes (calculado en 75 mil), el efecto se revierte aumentando el número de empresas certificadas.

De la Figura 5 se puede inferir que el umbral está vinculado al número de empresas certificadas en una ciudad, es decir, cuando en una ciudad este número es pequeño, los migrantes calificados causan que haya menos empresas certificadas, posiblemente porque coadyuvan a la generación de nuevas empresas más que a la certificación de ellas. En el mismo sentido, con base en la Figura 4 se puede inferir que el umbral está vinculado al tamaño de la ciudad, sugiriendo entonces que en una ciudad pequeña los migrantes calificados inciden primordialmente en la creación de nuevas empresas más que en la certificación de las existentes. Bajo este razonamiento, el isomorfismo empresarial manifestado en la certificación ISO 9000 e ISO 14000 se ve afectado por la migración de fuerza laboral calificada (mecanismo normativo); pero este isomorfismo debe concebirse como un proceso de largo plazo afectado a la vez por otros mecanismos de isomorfismo coercitivos, normativos y miméticos.

\section{Conclusiones}

En el presente estudio se aborda de manera empírica el argumento teórico de que las organizaciones, como sistemas abiertos, son afectadas por el ambiente (y viceversa) a través de la migración de trabajo calificado. Específicamente, se parte por una parte de que el ambiente induce a las organizaciones a alcanzar una homogeneización al ser presionadas por mecanismos ambientales, y por otra, que la migración de trabajo calificado (como elemento ambiental) está fuertemente vinculado a la empresa y su búsqueda de una mayor competitividad.

Así, se abordaron dos indicadores que de acuerdo a la teoría expuesta por Powell y Di Maggio (1999) pueden reflejar el proceso de homogeneización o isomorfismo organizacional de los sectores empresariales de las ciudades mexicanas: el primero, relacionado al isomorfismo competitivo, es el de diversidad de sectores económicos en una ciudad, el cual se establece bajo el argumento de que las ventajas competitivas inducen el desarrollo de clusters o grupos especializados de empresas, lo cual reduce el número de clases de sectores económicos. El segundo indicador, relacionado al isomorfismo institucional, es el de empresas certificadas en calidad, el cual se establece bajo el argumento de que las empresas buscan "mantenerse en el juego", es decir, sostenerse al menos en el nivel de calidad que les permitan competir frente a las demás empresas, y para lo cual obtienen las certificaciones.

El análisis empírico basado en una metodología conveniente para el estudio de la migración de trabajo calificado y la teoría organizacional ambiental considera la potencial endogeneidad de la migración mediante el uso de variables instrumentales para obtener estimadores consistentes de los mecanismos de isomorfismo. Utilizando otros mecanismos de isomorfismo de tipo normativo, mimético y coercitivo como variables de control, la evidencia sugiere que el efecto de la migración de trabajo calificado incide tanto en la disminución de los sectores económicos como en la certificación en calidad de las empresas, es decir, se encontró evidencia de que existe un efecto causal de la fuerza laboral migrante hacia el sector empresarial de las ciudades mexicanas. Con ambos indicadores se encontró que la relación entre el isomorfismo y 
la migración de trabajo calificado no es lineal, sino que los niveles o proporción de migrantes calificados requieren sobrepasar un umbral para incidir en el isomorfismo; por tanto, en este caso la migración de trabajo calificado es un mecanismo que interviene en el isomorfismo en el largo plazo.

Aunque los resultados sugieren que la migración de trabajo calificado induce un mayor isomorfismo competitivo e institucional en los sectores empresariales de las economías mexicanas, es conveniente realizar este análisis en periodos de tiempo más largos así como utilizar más indicadores de isomorfismo para robustecer los hallazgos de este estudio.

\section{Referencias}

Airola, J. (2007). The use of remittance income in Mexico. International Migration Review, 41(4), 850-859. https:// doi.org/10.1111/j.1747-7379.2007.00111.x

Anam, M. \& S.-H. Chiang (2007). Rural-urban migration of family labor: a portfolio model. Journal of International Trade and Economic Development, 16(3), 325-335. https://doi.org/10.1080/09638190701526477

Bermúdez Rico, R. E. (2010). Migración calificada e integración en las sociedades de destino. Sociedad y Economía, julio-diciembre (19), 135-150. Disponible en: http://sociedadyeconomia.univalle.edu.co/index.php/sociedad_y_ economia/article/view/4102

Borges, D.E., L.C. Dutra \& F.L. Scherer (2014). Meio ambiente e estratégia: um estudo multicaso no setor vitivinícola da região central do Rio Grande sob a perspectiva da teoria institucional. Revista de Administração da UFSM, septiembre (7), 40-54. https://doi.org/10.5902/1983465911358

Boustan, L., P. Fishback \& S. Kantor (2010). The effect of internal migration on local labor markets: american cities during the Great Depression. Journal of Labor Economics, 28(4), 719-746. https://doi.org/10.1086/653488

Burgelman, R. A. (2015). Prigogine's theory of the dynamics of far-from-equilibrium systems: application to strategic entrepeneurship and innovation in organizational evolution. En C. E. Shalley, M. A. Hitt, \& J. Zhou (Eds.), The Oxford handbook of creativity, innovation and entrepeneurship. (pp. 433-444). New York: Oxford University Press. https://doi.org/10.1093/oxfordhb/9780199927678.013.0029

Cabrera Rubio, A.L. (2016). La responsabilidad social empresarial de grandes multinacionales estadounidenses en México y su adaptación local desde la perspectiva institucional. Revista del Colegio de San Luis Nueva Época, 6(12), 116-158. http://dx.doi.org/10.21696/rcs16122016626

Cambell, A.B. (1993). Applied chaos theory. A paradigm for complexity. California: Academic Press.

Cameron, A.C. \& P.K. Trivedi (2010). Microeconometrics using STATA. (Revised Edition). Texas: Stata Corp.

Castelhano, M., C.Y.C.L. Lawell, D.A. Sumner \& J.E. Taylor (2016). The effects of migration and remittances on productive investment in rural Mexico, Working Paper, University of California at Davis. Disponible en: http:// www.des.ucdavis.edu/faculty/Lin

Chen, X., K. Frank, D. Thomas, \& L. Jianguo (2012). Weak ties, labor migration, and environmental impacts: towards a sociology of sustainability. Organization \& Environment, 25(1), 3-24. https://doi.org/10.1177/1086026611436216

Cuong, N.V. \& D. Mont (2012). Economic impacts of international migration and remittances on household welfare in Vietnam. International Journal of Development, 11(2), 144-163. https://doi.org/10.1108/14468951211241137

Doadrio, L., M. Alvarado \& N. Carrera (2015). Reforma de la normativa contable española: análisis de su entramado institucional. Revista de Contabilidad, 18 (2) 200-216. http://doi.org/10.1016/j.rcsar.2015.02.002

Filho, M.C.F. \& J.W. Sousa (2013). Structuring of the açai pulp exporting agribusiness' organizational field. Revista Brasileira de Gestão de Negócios, 15(47), 169-185. https://doi.org/10.7819/rbgn.v15i47.1353

Fuentes, A. (2013). A vocation for industrial transformation: ideology, organizational isomorphism, and upgrading in the Guatemalan sugar industry. Studies in Comparative International Development, agosto (14), 1-63. http:// dx.doi.org/10.1007/s12116-013-9142-y 
Gallén, M.L. \& C. Peralta de Grado (2016). Información de responsabilidad social corporativa y teoría institucional. Intangible Capital, 12(4), 942-977. http://dx.doi.org/10.3926/ic.732

Gellers, J. (2012). Greening constitutions with environmental rights: testing the isomorphism thesis. Review of Policy Research, 29 (4), 523-543. http://doi.org/10.1111/j.1541-1338.2012.00574.x

Goldin, I., K. Reinert, \& A.L. Beath (2007) La migración. En I. Goldin \& K. Reinert. Globalización para el desarrollo. (pp. 215-265). Bogotá: Editorial Planeta Colombiana y Banco Mundial. https://doi.org/10.1596/978-9-5842-1514-7

González Zepeda, C.A. \& L. Escala Rabadán (2014). Modelos organizativos e isomorfismo institucional entre asociaciones de migrantes michoacanos en Los Ángeles, California. Migración y Desarrollo, 12(22), 91-122. Disponible en: http://rimd.reduaz.mx/revista/rev22/4.pdf

Hannan, M. \& J. Freeman. (1977) The population ecology of organizations. American Journal of Sociology, 82(5), 929-964. https://doi.org/10.1086/226424

Hjorth B., S. V. (2009). La industria maquiladora y la migración interna en México. Revista Gaceta Laboral, 15(1), 5-28. Disponible en: http://produccioncientificaluz.org/index.php/gaceta/article/view/3602

IMCO (2016). Competitividad Urbana 2016. Reelección municipal y rendición de cuentas. ¿cómo lograr el círculo virtuoso? Instituto Mexicano para la Competitividad. Disponible en: https://imco.org.mx/wp-content/ uploads/2016/09/2016-Indice_Competitividad_Urbana-Documento.pdf

Jacomossi, F.A., R.M. Casagrande \& L.G. dos Reis (2015). O isomorfismo nos relatórios de sustentabilidade: uma análise das empresas brasileiras que compõem o Dow Jones Sustainability Index. Revista de Gestão Ambiental e Sustentabilidade, 4(2), 49-64. https://doi.org/10.5585/geas.v4i2.176

Lara Gómes, G. \& F. Pérez Sosa (2015). Determinantes del isomorfismo institucional de las sociedades cooperativas de ahorro y préstamo en México. Revista de Estudios Cooperativos, septiembre-diciembre (119), 77-106. http:// dx.doi.org/10.5209/rev_REVE.2015.n119.49068

Manic, M. (2016). The impact of remittances on regional consumption and investment. Journal of Regional Science. https://doi.org/10.1111/jors.12282.

Massey, D.S. (2015). A missing element in migration theories. Migration Letters, 12(3), 279-299. Disponible en: http://www.tplondon.com/journal/index.php/ml/article/view/568

Miluka, J., G. Carletto, B. Davis \& A. Zezza (2007). The vanishing farms? The impact of international migration on Albanian family farming. Paper prepared for presentation at the I Mediterranean Conference of Agro-Food Social Scientists. 103rd EAAE Seminar. Barcelona, Spain. https://doi.org/10.1596/1813-9450-4367

Mintzberg, H. (1997). Estructuras, fuerzas y formas de las organizaciones eficaces. En H. Mintzberg, J. B. Quinn, y J. Voyer. El proceso estratégico: conceptos, contextos y casos. (pp. 159-183). México: Pearson Education.

Mintzberg, H., H. Ahlstrand, \& J. Lampel (1998). Strategy Safari. The Free Press.

Mir, M. \& S.K. Bala (2015). NGO accountability in Bangladesh: two contrasting cases. Journal of Voluntary and Nonprofit Organizations, octubre (26), 1831-1851. http://doi.org/10.1007/s11266-014-9513-7

Mora Rivera, J.J. \& J. Arellano González (2016). Remittances as expenditure drivers in rural Mexico. Estudios Fronterizos Nueva Época, 17(33), 231-259. Disponible en: http://ref.uabc.mx/ojs/index.php/ref/article/view/569

Morais, D.O.C., N.Q.S. Oliveira \& E.M. Souza (2014). As práticas de sustentabilidade ambiental e suas influências na nova formatação institucional das organizações. Revista de Gestão Ambiental e Sustentabilidade, 3(3), 90-106.

Morgan, G. (2006). Images of Organization. Sage Publications. https://doi.org/10.5585/geas.v3i3.108

Moura, A.A.F., S.R.M. Domingos, A.C.A. Cabral, \& S.M. Santos (2014). Corporate Governance under the perspective of isomorphism: a BM\&FBovespa Banking Industry Approach. Revista de Gestão, Finanças e Contabilidade, 4(3), 23-44. https://doi.org/10.18028/2238-5320/rgfc.v4n3p23-44

Nava-Tablada, M. E. (2012). Migración internacional y cafeticultura en Veracruz, México. Migraciones internacionales, 6(3), 139-171. http://dx.doi.org/10.17428/rmi.v6i22.742

Ortega, F. \& G. Peri (2009). The causes and effects of international migrations: evidence from OECD countries 19802005. NBER Working Paper 14833. https://doi.org/10.3386/w14833

Pekkala Kerr, S. \& W. R. Kerr (2013). Immigration and employer transitions for STEM workers. American Economic Review, 103(3), 193-197. https://doi.org/10.1257/aer.103.3.193

Peri, G. (2012). The effect of immigration on productivity: evidence from US states. The Review of Economics and Statistics, 94(1), 348-358. https://doi.org/10.1162/REST_a_00137 
Piore, M.J. (1979). Birds of passage: migrant labor and industrial societies. Cambridge University Press. https://doi. org/10.1017/CBO9780511572210

Powell, W, \& P. Di Maggio (1999). Retorno a la jaula de hierro: el isomorfismo institucional y la racionalidad colectiva en los campos organizacionales. En P. Di Maggio \& W. Powell. El nuevo institucionalismo en el análisis organizacional. (pp. 104-125). México: Fondo de Cultura Económica.

Rivas Tovar, L.A. (2009). Evolución de la teoría de la organización. Universidad \& Empresa, julio-diciembre (17), 11-32. Disponible en: http://www.redalyc.org/pdf/1872/187214467001.pdf

Rivera, L. \& R. González (2016). Autonomía de gestión de la escuela. Las señales del isomorfismo empresarial. Voces de la Educación, 1(2), 93-101. Disponible en: http://www.revista.vocesdelaeducacion.com.mx/index.php/voces/ article/view/26/24

Rodríguez Gómez, R. (2009). Migración de personal altamente calificado de México a Estados Unidos: una exploración del fenómeno. Revista Electrónica de Investigación Educativa, 11(2), 1-34. Disponible en: http://www. redalyc.org/articulo.oa?id=15512151005

Rosas, A.J.S., K.L. Sánchez \& C.C. Martínez (2012). El agrupamiento empresarial de Jalcomulco, Veracruz: la institucionalización de un modelo de negocio en el turismo de aventura en México. Revista Ciencia Administrativa, 2(1), 25-36. Disponible en: http://revistas.uv.mx/index.php/cadmiva/article/view/1693

Ruiz Ortega, R. (2015). Convergencia de política hacia la gestión integral de recursos hídricos en México. Revista Mexicana de Análisis Político y Administración Pública, 4(2), 67-88. Disponible en: http://www.remap.ugto.mx/ index.php/remap/article/view/132/114

Salas, M. (2010). Isomorfismo en los programas de lucha contra la pobreza. Los programas Oportunidades y Puente. Desafíos, 22(1), 57-101. Disponible en: http://revistas.urosario.edu.co/index.php/desafios/article/view/1290

Sánchez Ramos, M.A. (2007). Tendencia hacia el isomorfismo en la administración pública municipal del Estado de México. Espacios Públicos, 10(20), 107-161. Disponible en: http://hdl.handle.net/20.500.11799/39632

Santermer, A. \& G. Giangaspero (2011). La venta cruzada a los clientes de remesas: resultados de un estudio sobre instituciones microfinancieras en los países andinos. Migraciones Internacionales, 6(2), 273-287. http://dx.doi. org/10.17428/rmi.v6i21.760

Stark, O. \& D.E. Bloom (1985). The new economics of labor migration. American Economic Review, 75(2), $173-178$. Disponible en: http://www.jstor.org/stable/1805591

Tovar C., L. M. \& J. S. Vélez V. (2007). Los efectos de la migración internacional en las condiciones de vida de los hogares colombianos. Desarrollo y Sociedad, Julio-diciembre (60), 155-197. DOI: 10.13043/dys.60.5

Wooldridge, J. M. (2009). Introductory econometrics. A modern approach. (4a Ed). Ohio: South-Western Cengage Learning.

Zarate-Hoyos, G.A. (2004) Consumption and remittances in migrant households: toward a productive use of remittances. Contemporary Economic Policy, 22(4), 555-565. https://doi.org/10.1093/cep/byh042 